\title{
Análise da Aplicação de Games User Research à Avaliação de Jogos Baseados em Localização
}

\author{
Nayana Carneiro \\ Universidade Federal do Ceará \\ Fortaleza, Brasil \\ nayanacarneiro@great.ufc.br
}

\author{
Ticianne Darin \\ Universidade Federal do Ceará \\ Fortaleza, Brasil \\ ticianne@virtual.ufc.br
}

\author{
Windson Viana \\ Universidade Federal do Ceará \\ Fortaleza, Brasil \\ windson@great.ufc.br
}

\begin{abstract}
RESUMO
Os jogos pervasivos oferecerem aos jogadores uma experiência adaptável e que mistura os mundos real e virtual. Como subtipo desses jogos, têm-se os Jogos Baseados em Localização (JBL), que incorporam a posição física do jogador às suas regras e dinâmica. Esses jogos possuem características peculiares (como o uso de informações contextuais, persistência e forte apelo à interação social) e, por isso, avaliá-los adequadamente, de modo a garantir seu bom funcionamento e a satisfação de seus usuários, se torna um desafio. O presente trabalho tem por objetivo avaliar se métodos de avaliação usados em Games User Research (GUR) podem atender às características e necessidades específicas dos JBL, visando contribuir para a discussão e construção de um conjunto de métodos adequados a esse domínio.
\end{abstract}

\section{Palavras-chave}

Jogos pervasivos; Jogos baseados em localização; Métodos de avaliação; Interação; Games User Research.

\section{ACM Classification Keywords}

H.5.2. User Interfaces: Evaluation/methodology; H.5.m. Information interfaces and presentation: Miscellaneous.

\section{INTRODUÇÃO}

Jogos digitais são atrativos por oferecerem acesso a mundos virtuais imersivos. Com a popularização e evolução das tecnologias móveis, ampliou-se a gama de dispositivos capazes de executar diversos tipos de jogos digitais e atingir variados públicos, tornando os dispositivos móveis uma das principais plataformas de jogos. Isso abriu espaço para a disseminação dos jogos pervasivos, que resultam da mistura entre os ambientes real e virtual, de forma que os limites espaciais ou temporais entre ambos não mais existam [13]. Os jogos pervasivos modernos podem enriquecer o seu gameplay usando dados contextuais do jogador, obtidos pelos sensores de seu smartphone, para entregar uma

\footnotetext{
Permission to make digital or hard copies of all or part of this work for personal or classroom use is granted without fee provided that copies are not made or distributed for profit or commercial advantage and that copies bear this notice and the full citation on the first page. Copyrights for components of this work owned by others than the author(s) must be honored. Abstracting with credit is permitted. To copy otherwise, or republish, to post on servers or to redistribute to lists, requires prior specific permission and/or a fee. Copyright 2018 SBC.
}

IHC 2018, Anais Estendidos do XVII Simpósio Brasileiro sobre Fatores Humanos em Sistemas Computacionais Outubro 22-26, 2018, Belém, Brasil WTD-IHC experiência de jogo adaptável ao local onde ele se encontra, ao que faz, ou mesmo ao que sente [6]. Os Jogos Baseados em Localização (JBL) são jogos pervasivos que usam tecnologias de localização para incorporar a posição física do jogador às suas regras e dinâmicas [14]. Nos últimos anos, alguns JBL alcançaram grande sucesso entre 0 público, como é o caso de Ingress (Niantic, 2012) e Pokémon GO (Niantic, 2016), e conquistaram espaço não só na indústria de jogos, mas também despertaram interesse na comunidade acadêmica. Isso é motivado, dentre outros fatores, pelo potencial de aplicação desses jogos em diversas áreas (como turismo, saúde e educação) e pelos diversos desafios de pesquisa que os acompanham, como a transposição de mapas entre cidades, o uso da localização no game design, a segurança dos jogadores e questões de ética e privacidade.

Porém, devido às características peculiares desses jogos (e.g. persistência, uso de informações contextuais, necessidade de deslocamento e forte apelo à interação social), avaliar a interação do usuário com um JBL se torna um desafio. É necessário considerar questões técnicas, mas também explorar a perspectiva do jogador e seu contexto de uso, de forma a captar aspectos que reflitam sua relação com o jogo, sua satisfação, motivação e o cumprimento de suas expectativas [10].

Nesse sentido, Games User Research (GUR) oferece um conjunto de técnicas e ferramentas específicas para avaliar a experiência proporcionada por jogos digitais e obter insights sobre o comportamento dos usuários que permitam melhorar o design de jogos digitais em geral [12]. Assim, o objetivo desta pesquisa é avaliar se métodos utilizados pela GUR podem atender às necessidades específicas de avaliação da interação de JBL. Dessa forma, espera-se contribuir para a discussão e estabelecimento de um conjunto de direcionamentos para a avaliação da Interação Humano-Computador (IHC) nesse domínio.

\section{FUNDAMENTAÇÃO TEÓRICA}

Nesta seção são apresentados conceitos relacionados a esta pesquisa, compondo uma visão geral sobre JBL e GUR.

\section{Jogos Baseados em Localização}

Atualmente, os jogos baseados em localização são entendidos como jogos pervasivos que usam tecnologias de localização para incorporar a posição física do jogador às suas regras e dinâmicas [14]. Essas informações são usadas para modificar o estado do jogo em sua execução e, com 
isso, criar uma conexão entre o mundo virtual e o mundo real, usando o espaço físico como cenário. Esses jogos promovem uma "percepção dupla" do espaço, estabelecendo entre o mundo físico e o virtual uma relação significante para a condução do jogo [4].

Além disso, é uma característica essencial dos JBL requerer que os jogadores se desloquem fisicamente no mundo real para atingir seus objetivos no jogo. Esses deslocamentos (que podem ser tomados de posição absoluta ou relativa) servem a propósitos diversos na dinâmica do jogo, como coletar itens, explorar determinados cenários e encontrar personagens em uma missão [20]. Para tal, os jogadores usam dispositivos com sensores de localização enquanto se movem por ambientes reais e visitam pontos de interesse. As interações ocorrem, então, enquanto os jogadores exploram o mundo. Para isso, os JBL usam tecnologias sensíveis à localização [3] e dependem de dispositivos portáteis, como PDAs, receptores GPS e smartphones. Além disso, os JBL podem usar uma gama de redes sem fio, como Wi-Fi, Bluetooth e $3 \mathrm{G} / 4 \mathrm{G}$, para realizar a comunicação entre os espaços físicos e os digitais e entre os jogadores [2]. Outras características comuns aos JBL são: gameplay independente de tempo ou lugar específico (i.e., o jogador não precisa estar em um ambiente específico ou esperar por determinado horário para jogar), forte apoio na interação social para progresso no jogo e a possibilidade de jogar enquanto realiza outras atividades $[13,20]$.

Assim, as pesquisas com JBL envolvem diversos temas e desafios, como game design [1], desenvolvimento de ferramentas de autoria [8], balanceamento de mapas e transposição [9], e avaliações da $\operatorname{IHC}[6,15,16]$ - principal ponto de interesse deste trabalho.

\section{IHC e Games User Research}

Games User Research é um campo de estudo emergente que une Interação Humano-Computador, Desenvolvimento de Jogos e Psicologia Experimental [19], ocupando-se em estudar a relação entre o usuário e o jogo. GUR é um termo guarda-chuva para as áreas que tratam desse estudo. Assim, GUR é um campo interdisciplinar de prática e pesquisa que busca melhorar a qualidade da usabilidade e da experiência dos usuários em jogos digitais. Qualquer aspecto que influencie a percepção e a experiência do usuário é uma questão a ser investigada pela GUR [5], que procura compreender as motivações dos jogadores, explicar e prever suas ações, além de buscar novas formas de coletar dados sobre os usuários e aplicá-los no design de jogos. Assim, GUR é uma parte central no desenvolvimento de jogos, pois ajuda os game designers a alcançarem suas metas, permitindo-lhes averiguar se a experiência planejada está sendo de fato entregue aos jogadores [5].

Para alcançar tal compreensão, a GUR se utiliza de processos de avaliação que permitam observar os usuários enquanto interagem com o jogo e seus componentes, e coletar dados significantes para a análise e compreensão da interação e da experiência com o jogo. Como na tradicional avaliação de IHC, o objetivo não é avaliar o usuário, mas obter insumos para melhorar todos os aspectos do game design, tendo como guia as evidências empíricas advindas da experimentação e da aplicação de diversos testes. Assim, os estudos de GUR compreendem uma coleção de métodos que permitem ao game designer aproximar sua criação da experiência que será vivenciada pelo jogador [11].

No entanto, identificar quais são os melhores métodos para se obter tais insumos ainda é um desafio, dada a natureza intrincada e subjetiva dos jogos e os diversos aspectos que permeiam a experiência de um usuário com um produto interativo. Apesar dos métodos tradicionais de avaliação terem feito progresso na compreensão da usabilidade de aplicações e sistemas de produtividade, as características específicas dos jogos implicam que muitos desses métodos não podem ser aplicados do mesmo modo para avaliar jogos. Um exemplo claro disso é a presença da frustração na interação, que é naturalmente permitida como parte de um jogo, mas não é sequer tolerada em sistemas de produtividade. Desse modo, o aperfeiçoamento de métodos tradicionais de avaliação da IHC para a prática de GUR é um tópico importante para a comunidade de $\mathrm{IHC}[5,10]$.

\section{OBJETIVO E RESULTADOS ESPERADOS}

JBL e GUR são domínios relativamente novos, cujas bases de conhecimento ainda estão em construção, o que abre diversas oportunidades de pesquisa. É sabido que a avaliação de IHC é crucial para garantir que o usuário tenha suas necessidades e expectativas atendidas [18]. No tocante aos JBL, torna-se ainda mais valiosa, uma vez que questões de usabilidade - que podem ser pouco impactantes em alguns sistemas de produtividade ou outros tipos de jogos podem causar grandes impactos negativos na experiência e até mesmo colocar em risco os jogadores, que estão mais expostos neste contexto [15].

Diante disso, o principal objetivo desta pesquisa é avaliar se métodos utilizados pela Games User Research podem atender às necessidades específicas de avaliação da interação de jogos baseados em localização. Pretende-se investigar se esses métodos são capazes de compreender as características específicas de JBL.

Como resultados, esta pesquisa visa estabelecer um conjunto de direcionamentos para a avaliação com usuários nesse domínio. Assim, espera-se obter dois produtos intermediários e um produto principal. Os intermediários são: (1) um panorama da avaliação de IHC em JBL; e (2) um estudo de caso sobre a aplicabilidade de métodos da GUR para avaliar JBL. A natureza do produto principal será definida com base nas lacunas detectadas ao longo da condução da pesquisa, de acordo com os resultados obtidos na elaboração dos produtos intermediários. No entanto, é possível que tal produto seja (1) um instrumento de avaliação específico para JBL - caso as etapas anteriores revelem que os métodos existentes não são eficazes para este contexto, ou (2) um conjunto de guidelines para a utilização de métodos já aplicados em GUR na avaliação de 
JBL, por exemplo. De modo geral, pretende-se gerar uma discussão acerca da avaliação desse tipo de jogo, visando detectar desafios na área e estabelecer caminhos que a comunidade de IHC poderia seguir nos próximos anos de pesquisa.

\section{METODOLOGIA}

Para alcançar tais resultados, a pesquisa será dividida em quatro etapas, conforme mostra a Figura 1. Vale ressaltar que os métodos e estratégias de avaliação que serão utilizados nos próximos passos da pesquisa, por enquanto, não estão definidos, pois os resultados de cada etapa ajudarão a definir as diretrizes para a etapa seguinte.

\section{Mapeamento sistemático da literatura}

Atualmente em andamento, esta etapa consiste na condução de um mapeamento sistemático da literatura [7, 17], para aprofundar o conhecimento do estado da arte em avaliação de JBL. O objetivo é identificar quais métodos e técnicas são mais utilizados para avaliar JBL e quais aspectos da interação são explorados (e.g., usabilidade, jogabilidade, diversão, experiência), além de outras informações que possam ajudar a estabelecer os rumos das etapas seguintes.

\section{Estudo de caso}

Com base nos resultados do mapeamento sistemático, será definido um conjunto de métodos que serão utilizados na avaliação de, pelo menos, um JBL disponível no mercado, bem como um conjunto de aspectos da interação a serem avaliados. Os resultados obtidos darão insumos para estabelecer a direção que será seguida na etapa 3 e apontarão lacunas que poderão ser supridas pela pesquisa.

\section{Elaboração do produto principal}

Com base nos resultados obtidos nas etapas 1 e 2 da pesquisa será definido o produto principal. No entanto, são previstas, inicialmente, duas possibilidades: (1) a criação de um instrumento de avaliação específico para JBL, ou (2) a elaboração de um conjunto de guidelines que organize e oriente a condução de uma avaliação de IHC de um JBL, aplicando métodos e instrumentos detectados na literatura. De acordo com o tipo de produto escolhido, será utilizada uma metodologia adequada para alcançar o resultado esperado.

\section{Avaliação da solução proposta}

Após a elaboração do produto, este será avaliado para averiguar se as expectativas e a contribuição pretendidas foram alcançadas. De acordo com o tipo de produto gerado, a avaliação poderá seguir uma das duas abordagens: (1) validação com especialistas em desenvolvimento de jogos; ou (2) uso do produto em avaliações com usuários reais. Assim como na etapa anterior, a metodologia será escolhida após a delimitação do produto principal.

\section{RESULTADOS PARCIAIS}

$\mathrm{Na}$ condução do mapeamento sistemático (em andamento), a busca por trabalhos foi realizada em cinco bases (Scopus, ACM, Web of Science, Science Direct e IEEE Xplore), utilizando a string (("location based game" OR "location based mobile game") OR (("pervasive game" OR "ubiquitous game" OR "augmented reality game") AND (location OR locative OR mobile OR outdoor))) AND ((user OR player OR gamer) AND (evaluation OR assessment $O R$ study)). Foram obtidos 437 trabalhos, dos quais 381 foram excluídos por não atenderem aos critérios de inclusão estabelecidos, o que resulta em 31 trabalhos aceitos até o momento.

A seleção dos estudos para leitura completa ainda está em andamento, mas em uma análise preliminar desses trabalhos é possível perceber grande variedade de métodos e instrumentos empregados na avaliação dos jogos, sendo frequente a utilização de questionários ad hoc, elaborados para atender interesses específicos dos avaliadores ou adaptados de instrumentos tradicionais para captar determinadas dimensões dos jogos estudados. Nesses trabalhos, há também diversidade nos aspectos da interação avaliados, que incluem usabilidade, jogabilidade, diversão, engajamento, imersão, experiência, impacto e aspectos comportamentais afetáveis pela utilização de JBL - muitos desses motivados pelo sucesso de Pokémon GO.

Ademais, muitos trabalhos apresentam o design de um JBL acompanhado de uma avaliação para averiguar o potencial de atendimento a propósitos específicos, que podem ser relacionados a saúde, educação, cultura e questões técnicas do desenvolvimento desses jogos. De modo geral, essas percepções parecem apontar para a inexistência de um conjunto de métodos específicos para avaliar esses jogos ou mesmo de guidelines que orientem a avaliação.

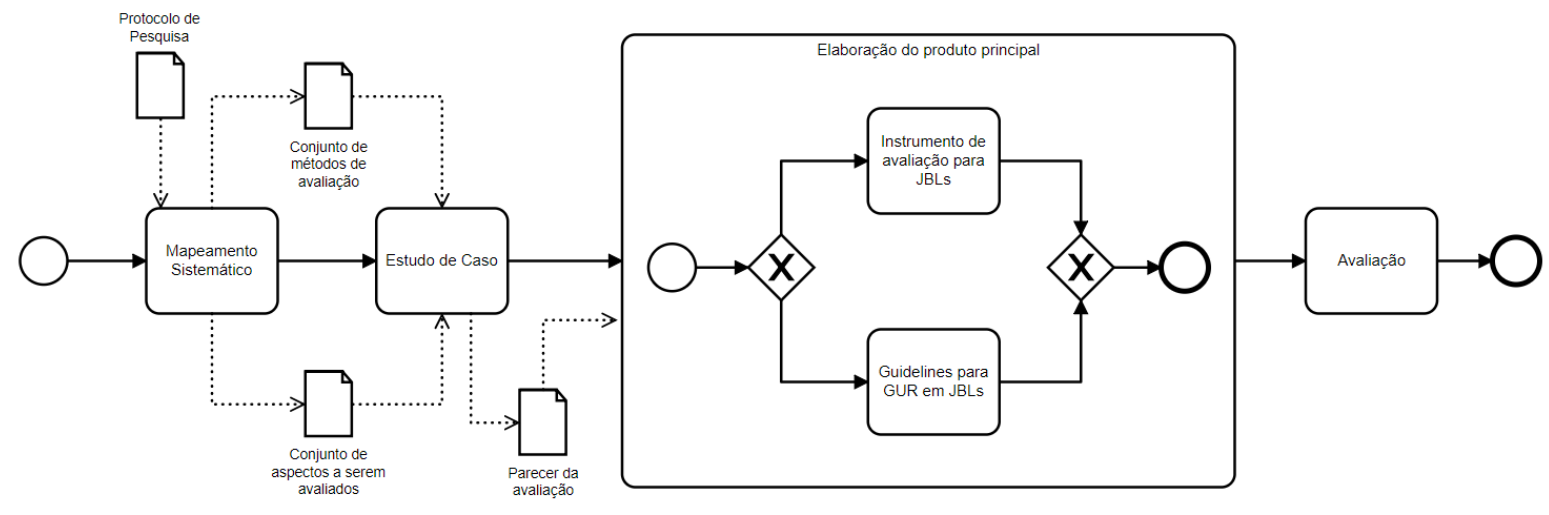

Figura 1. Etapas planejadas para a condução do trabalho 


\section{CONSIDERAÇÕES FINAIS}

A pesquisa aqui apresentada busca avaliar se métodos utilizados pela Games User Research podem atender às necessidades específicas de avaliação da interação de jogos baseados em localização. Como contribuição, espera-se compor um panorama dos estudos em avaliação da IHC em JBL, e atuar no suprimento de lacunas detectadas ao longo da pesquisa, por meio da aplicação de conhecimentos provenientes da GUR. Ademais, ao constatar como a GUR pode ser empregada para dar subsídio a avaliações mais efetivas de JBL, desenvolvedores de jogos e a comunidade de IHC interessada no domínio serão beneficiados.

\section{AGRADECIMENTOS}

Agradecimentos à Fundação Cearense de Pesquisa e Cultura (FCPC) pela bolsa concedida a Nayana Carneiro, financiada com recursos da Lei de Informática.

\section{REFERÊNCIAS}

1. Anne Bowser, Derek Hansen, Jocelyn Raphael, Matthew Reid, Ryan Gamett, Yurong He, Dana Rotman and Jenny Preece. 2013. Prototyping in PLACE: a scalable approach to developing locationbased apps and games. In Proceedings of the SIGCHI Conference on Human Factors in Computing Systems (pp. 1519-1528). ACM.

2. Michiel de Lange. 2009. From always on to always there: Locative media as playful technologies. Digital cityscapes: Merging digital and urban playspaces. New York: Peter Lang, 55-70.

3. Adriana de S. Silva e Daniel Sutko. 2009. Digital cityscapes: Merging digital and urban playspaces. Peter Lang.

4. Adriana de Souza e Silva e Daniel M. Sutko. 2011. Theorizing locative technologies through philosophies of the virtual. Communication Theory, 21(1):23-42.

5. Anders Drachen, Mirza-Babaei Pejman, and Lennart E. Nacke. 2018. Games User Research. Oxford U. Press.

6. Vlasios Kasapakis and Damianos Gavalas. 2015. Pervasive gaming: Status, trends and design principles. Journal of Network and Computer Applications, 55, 213-236.

7. Barbara Kitchenham, Pearl Brereton, David Budgen, Mark Turner, John Bailey, and Stephen Linkman. 2009. Systematic literature reviews in software engineering - A systematic literature review. Information and software technology, 51(1), 7-15.

8. Luís Maia, Carleandro Nolêto, Messias Lima, Cristiane Ferreira, Cláudia Marinho, Windson Viana, and Fernando Trinta. 2017. Lagarto: A location-based games authoring tool enhanced with augmented reality features. Entertainment Computing, 22, 3-13.

9. Luís Maia, Windson Viana, and Fernando Trinta. 2017. Using Monte Carlo tree search and google maps to improve game balancing in location-based games. In Computational Intelligence and Games. IEEE.

10. Lennart E. Nacke, Jörg Niesenhaus, Stephan Engl, Alessandro Canossa, Kai Kuikkaniemi and Thomas Immich. 2010. Bringing digital games to user research and user experience. Proceedings of the Entertainment Interfaces Track 2010 Interaktive Kulturen, 12-15.

11. Lennart E. Nacke. 2015. Games user research and physiological game evaluation. In Game user experience evaluation (pp. 63-86). Springer, Cham.

12. Lennart E. Nacke, Christiane Moser, Anders Drachen, Pejman Mirza-Babaei, Andrea Abney and Zhu Zhenyu. 2016. Lightweight Games User Research for Indies and Non-Profit Organizations. In Ext. Abstracts of the CHI Conference on Human Factors in Computing Systems.

13. Eva Nieuwdorp. 2007. The pervasive discourse: an analysis. Computers in Entertainment (CIE), 5(2), 13.

14. Carleandro Nôleto, Windson Viana, Fernando Trinta. 2017. Ferramenta de Autoria para o Desenvolvimento de Jogos Pervasivos Baseados em Real. Aumentada.

15. Janne Paavilainen, Hannu Korhonen, Kati Alha, Jaakko Stenros, Elina Koskinen, and Frans Mayra. 2017. The Pokémon GO experience: A location-based augmented reality mobile game goes mainstream. In Proceedings of the CHI Conference on Human Factors in Computing Systems (pp. 2493-2498). ACM.

16. Alison R. Panisson, Daniela Schmidt, Regio Michelin, Rubens P. Gonçalves, and Milene Silveira. 2015. Challenges for evaluating mobile systems with moving users. In Proceedings of the 14th Brazilian Symposium on Human Factors in Computing Systems (IHC'15), Artur Henrique Kronbauer, Ecivaldo Mattos, Andreia Libório Sampaio, and Clodis Boscarioli (Eds.). ACM, New York, NY, USA, Article 52, 4 pages.

17. Kai Petersen, Sairam Vakkalanka, and Ludwik Kuzniarz. 2015. Guidelines for conducting systematic mapping studies in software engineering: An update. Information and Software Technology 64: 1-18.

18. Jenny Preece, Helen Sharp, and Yvonne Rogers. 2015. Interaction Design: Beyond Human-Computer Interaction. John Wiley \& Sons.

19. Magy Seif El-Nasr, Heather Desurvire, Lennart E. Nacke, Anders Drachen, Licia Calvi, Katherine Isbister, and Regina Bernhaupt. 2012. Game user research. In CHI'12 Extended Abstracts on Human Factors in Computing Systems (pp. 2679-2682). ACM.

20. Luis Silva. 2016. Jogos Baseados em Localização: Características, Limitações e Desafios. Monografia de Qualificação de Doutorado. Universidade Federal do Ceará, Fortaleza, CE. 